\title{
Banisterine Alleviates Morphine-based Nephrotoxicity by Antioxidant Property: An In vivo Study
}

\author{
Mohammad Reza Salahshoor, Cyrus Jalili', Amir Abdolmaleki, Shiva Roshankhah
}

Department of Anatomical Sciences, Medical School, Kermanshah University of Medical Sciences, 'Department of Anatomical Sciences, Medical Biology Research Center, Kermanshah University of Medical Sciences, Kermanshah, Iran

\begin{abstract}
Background: Banisterine (BAN) as an alkaloid agent has antioxidant properties. The morphine (MOR) with the character of free radical generation has an effective role on renal pathogenesis. Objectives: This investigation critically examines the effects of the BAN against MOR-induced damage to the kidneys of rats. Materials and Methods: Sixty-four male Wistar rats were randomly assorted into 8 groups (8 rats in each), including the saline (Sal), MOR, BAN $(5,10,15 \mathrm{mg} / \mathrm{kg})$ and MOR + BAN treatment groups. All experimental procedures were applied by intraperitoneal injection daily for 20 days. Whole investigated values consist of total animal weight, weight of kidney, morphological criteria of kidney, antioxidant capacity and serum nitrite oxide levels. Results: Intraperitoneally MOR application significantly increased the levels of renal Malondialdehyde (MDA), blood urea nitrogen (BUN), blood creatinine, and blood nitrite oxide and also reduced the glomerular number and tissue ferric reducing/antioxidant power (FRAP) level compared to the Sal control group $(P<0.05)$. Treatment of BAN and BAN + MOR in all doses significantly reduced the levels of BUN, MDA, creatinine, glomerular diameter, and nitrite oxide and also increased the glomerular number and tissue FRAP levels compared to the MOR group $(P<0.05)$. Conclusion: The findings support the idea that the BAN with its antioxidant nature can eliminate MOR renal toxicity.

Key words: Antioxidant, banisterine, in vivo, morphine, nephrotoxicity

\section{SUMMARY}

- Morphine (MOR) administration significantly increased the serum levels of the kidney Malondialdehyde, blood nitrite oxide, blood urea nitrogen and blood creatinine and decreased glomerular number and ferric reducing/antioxidant power level at the end of 20 days in MOR control group rats

- Banisterine (BAN) treatments had a significant effect on the improvement of kidney parameters in BAN and BAN + MOR group rats at the end of the 20 days

- BAN might be a good candidate for kidney treatment, especially improved kidney injury induced by MOR

- This finding is important due to the increased incidence of kidney injury due to the Painkillers drug containing morphine and addicts.
\end{abstract}

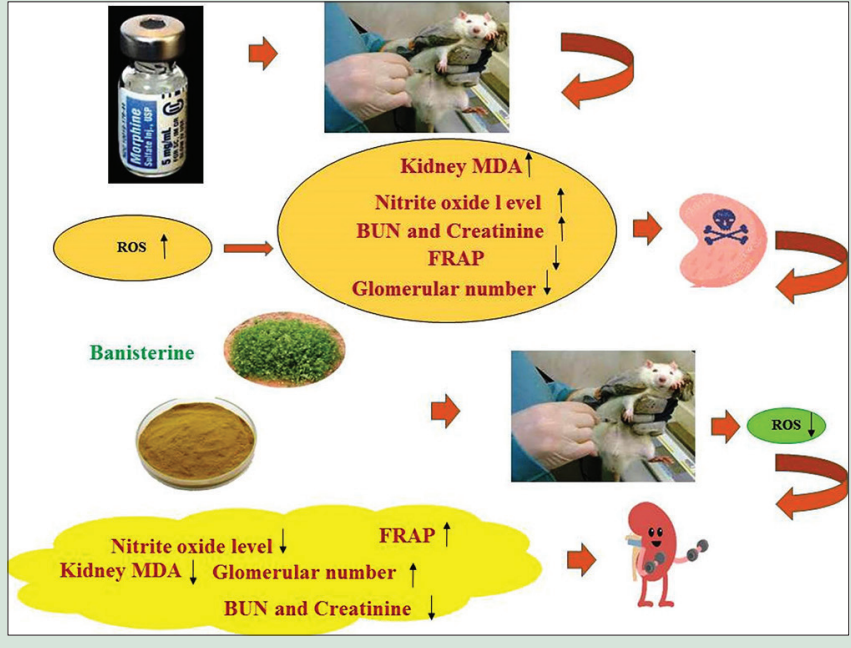

Abbreviations Used: BAN: Banisterine; MOR: Morphine; MDA: Malondialdehyde; BUN: Blood urea nitrogen; FRAP: Ferric reducing/antioxidant power; OD: Optical density; MAO: Monoamino oxidase.

\section{Correspondence:}

Dr. Shiva Roshankhah,

Department of Anatomical Sciences, Medical

School, Kermanshah University of Medical

Sciences, Daneshgah Ave., Taghbostan,

Kermanshah, Iran.

E-mail: roshankhah@yahoo.com

DOI: 10.4103/pr.pr_97_19

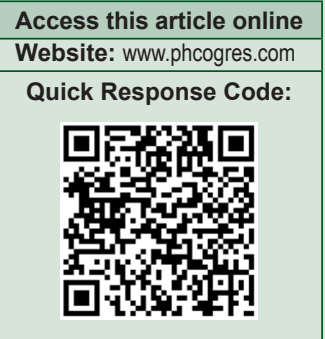

\section{INTRODUCTION}

Opioids are generally used for pain relief. Together with their activity, they also produce free radicals causing cellular apoptosis. Morphine (MOR) is a psychoactive chemical available in opium acts as analgesic drug. ${ }^{[1]}$ This drug has addictive effects, thus causes physiological dependence and also has the ability of oxidative stress production. ${ }^{[2]}$ Two significant pathological changes occur immediately after MOR injection, the increased concentration of oxidative stress and enhancement in the lipid oxidase activity. Subsequently, irreversible damage to the cell membrane can be seen. ${ }^{[3]}$

The high oxidative stress levels result in an imbalance between free radical and antioxidant productions. This unbalanced state causes the oxidation of biomolecules (they promote oxidation reactions with proteins, lipids and DNA and can thus be highly detrimental), changes in cell structure and function. ${ }^{[4]}$ Oxidative stress can deeply alteration such mitochondrial parameters. The oxidative harvests issuing from each biomolecule are multifaceted and manifold. Reactive oxygen species, coming from the reaction of nitric oxide and superoxide anion, are robust oxidants accomplished of destructive lipids, proteins and DNA. Reactivity, the mechanism of manufacture and the products formed vary dependent on the free radical (hydroxyl radical, superoxide anion, Peroxynitrite) and the molecular goal (cholesterol, aromatic, phospholipids, and aliphatic amino acids). ${ }^{[5]}$ Prolonged use of opioids is related to the aggregation of oxidative stress in the kidney. This destructive accumulation leads to the breakdown of nuclear DNA, denaturation of proteins and degeneration

This is an open access journal, and articles are distributed under the terms of the Creative Commons Attribution-NonCommercial-ShareAlike 4.0 License, which allows others to remix, tweak, and build upon the work non-commercially, as long as appropriate credit is given and the new creations are licensed under the identical terms.

For reprints contact: reprints@medknow.com

Cite this article as: Salahshoor MR, Jalili C, Abdolmaleki A, Roshankhah S. Banisterine alleviates morphine-based nephrotoxicity by antioxidant property: An in vivo study. Phcog Res 2020;12:137-42. 
of cell membrane. ${ }^{[6]}$ In such a situation, the renal damage will appear. ${ }^{[7]}$ The kidneys are the main blood purifier organs discharging toxins into urine. The process of toxins and drugs into the urinary system can be influenced by chronic renal disorders. ${ }^{[8]}$ MOR by detrimental renal damage causes an increase in albumin discharge and the emergence of proteinuria conditions. Chronic proteinuria will terminate to renal malfunction. ${ }^{[9]}$

The Peganum harmala is known as a member of the Zygophyllaceae family. ${ }^{[10]}$ It is cultivated in the vast geographical range from North Africa to the Middle East. The seeds and roots of $P$. harmala contain alkaloids. ${ }^{[11]}$ Banisterine (BAN) and harmaline are the most abundant alkaloids exist in $P$. harmala that have therapeutic effects. ${ }^{[12]}$ Totally portions of this plant are believed to be toxic and sever intoxication happens in national animals. Digestive and nervous syndromes have been detected in animals that eat sublethal amount of the plant. The toxicated animal appears in a narcotic state disturbed by occasional short dated of excitement. Harmine (BAN), C13H12ON2, It is existing in P. harmala. The alkaloid is optically inactive and forms colorless rhombic prisms from methanol. Pharmacologically, BAN bear a resemblance to harmaline in its activities however is less toxic. ${ }^{[13]}$ The essence of this plant can be used for the treatment of bradycardia, reduction in blood pressure, adjustment of angiogenesis. Along with their medicinal effects, they act as anti-allergic, anti-spasm and anti-adrenergic agent. ${ }^{[14]} P$. harmala has been used extensively in the field of traditional medicine, for example menstrual cycle regulator, food appetizer, annihilator of parasites. ${ }^{[15]} \mathrm{BAN}$ is an active component of $P$. harmala with alkaloid property as a member of the beta-carboline family. The BAN substance is derivate from $P$. harmala and is completely known as antioxidant. ${ }^{[16]}$ It is an active controller of tyrosine phosphorylation-regulated kinase (DYRK) enzyme, which is involved in the adjustment of mitosis evasion, apoptosis, transcription factors and pro-inflammatory cytokines. ${ }^{[17]}$ For this reason, in human tumor cells the BAN shows cytotoxic activity. ${ }^{[18]}$ Besides, it can suppress TNF- $\alpha$ activity and nitrite oxide production as oxidative stress agents. ${ }^{[19]}$ It has been found that the levels of MOR in the body lead to an increase in the level of nitrite oxide and oxidative stress. ${ }^{[20]}$ Various approaches have been suggested the toxic trace of MOR along with beneficial effects of BAN. ${ }^{[21]}$ There are not enough surveys on the therapeutic impact of BAN on renal diseases. Thus, this study will provide a clear attitude whether BAN alleviates the MOR-related renal toxicity by antioxidant pathways in animal male Wistar rats.

\section{MATERIALS AND METHODS}

\section{Experimental animals}

The present experiment was carried out in the Anatomy Department of the Kermanshah University of Medical Faculty from May 2018 to December 2018. A total of 64 male Wistar rats weighting 250-270 $\mathrm{g}$ and 8 weeks old were purchased from Pasteur Institute of Iran (IPI, Tehran, Iran). The nutritional and ideal living conditions for laboratory animals were provided as follows: plastic cages with access to water and food pletes freely, room temperature around $23^{\circ} \mathrm{C} \pm 2^{\circ} \mathrm{C}$, with a relative humidity of $50 \% \pm 5 \%$ and 12 day/12 night diurnal cycle. All experimental processes were conformed to the ethical and humane principles of research and also were approved by the Ethics Committee of Kermanshah University of Medical Sciences (ethics certificate no. 1395.38). ${ }^{[7]}$

\section{Study design and treatments}

All treatments were applied through intraperitoneal injection. Three types of treatment doses were prescribed. The MOR injection in which a single dose of $20 \mathrm{mg} / \mathrm{kg}$ for the first 5 days, a double dose of $20 \mathrm{mg} / \mathrm{kg}$ daily for the second 5 days and a double dose of $30 \mathrm{mg} / \mathrm{kg}$ daily for the remaining 10 days were administered. The BAN injections were 5, 10 and $15 \mathrm{mg} / \mathrm{kg}$ daily for 20 days. The saline (Sal) injection, which administered just for control group is equivalent to the amount of experimental groups. Eight random groups with 8 rats in each were selected. The groups were respectively included the Sal (control group), MOR group, BAN groups (three groups of 5, 10 and $15 \mathrm{mg} / \mathrm{kg}$ ), BAN + MOR groups (three groups of 5,10 and $15 \mathrm{mg} / \mathrm{kg}$ ). ${ }^{[1,22]}$

\section{Weight of rats, kidney and blood serum collection}

Total body and renal weight were measured by a microbalance (Precisa 125A; Switzerland) at the first and end of the study. In this process, animals were intraperitoneally anesthetized with a single dose of ketamine/xylazine $(100 / 10 \mathrm{mg} / \mathrm{kg})$. To collect the blood through the right ventricle, the thoracotomy procedure was done. The blood sample was aspirated and incubated for $15 \mathrm{~min}$ at $37^{\circ} \mathrm{C}$ to form clot. Then the centrifugation was applied at $3000 \mathrm{rpm}$ for $15 \mathrm{~min}$. The separated serum was stored at $-70^{\circ} \mathrm{C}$ for the measurement of the biochemical factors. ${ }^{[10]}$

\section{Histological and morphometric analysis}

A vertical incision was made on kidneys in order to the creation of two equal parts. The samples of kidneys were fixed by infiltration of $10 \%$ formalin solution for a week immersion. The conventional histological tissue process was run and $5 \mu \mathrm{m}$ serial sections were prepared (microtome, EC350-2). The staining was applied by Hematoxylin and Eosin. The diameter and number of glomeruli were examined by a microscope linked to a DP12 camera (Olympus BX-51T-32E01 with 3.34-million pixel resolution). The morphometric features were analyzed by Olysia Bio software (Olympus Optical; Japan)..$^{[7]}$

\section{Biochemical marker assays}

The concentrations of creatinine and blood urea nitrogen (BUN) exist in blood samples were analyzed biochemically (autoanalyzer, RA 1000; Technicon Instruments; USA). ${ }^{[23]}$

\section{Nitrite oxide assay}

In this technique, zinc sulfate powder was added to eliminate the proteins of blood serum. $6 \mathrm{mg}$ powder of zinc sulfate was mixed with $400 \mu \mathrm{l}$ of serum samples and centrifuged $(12,000 \mathrm{rpm}, 10 \mathrm{~min})$. The nitrite oxide available in supernatant was measured. The previous sample $(50 \mu \mathrm{l})$ was added to Griess reagent (100 $\mu \mathrm{l}$, Sigma; USA). Then, it was incubated (30 min at room temperature). The ELISA reader (Hyperion; USA) device measured the sample optical density at the wavelength of $450 \mathrm{~nm}^{[1]}$

\section{Biochemical techniques of ferric reducing/antioxidant power and malondialdehyde}

Based on the colorimetric analysis, the specious of thiobarbituric acid reactive were measured by the means of malondialdehyde (MDA) (last product of lipid peroxidation) in renal tissue to evaluate the levels of oxidative stress. The ferric reducing/antioxidant power (FRAP) technique was hired for the measurement of antioxidant capacity in kidney. $1.5 \mathrm{ml}$ of chloride ferric (Sigma, USA) and $30 \mathrm{ml}$ of acetate buffer (Sigma, USA) were available in FRAP substance. Serial concentrations of $\mathrm{FeSO}_{4} \cdot 7 \mathrm{H}_{2} \mathrm{O}$ (Sigma, USA) were considered as an external standard. ${ }^{[23]}$

\section{Statistical analysis}

The Kruskal-Wallis test was hired to examine data normality and homogeneity. Statistical comparisons were investigated via one-way analysis of variance, followed by the least significant difference post hoc 


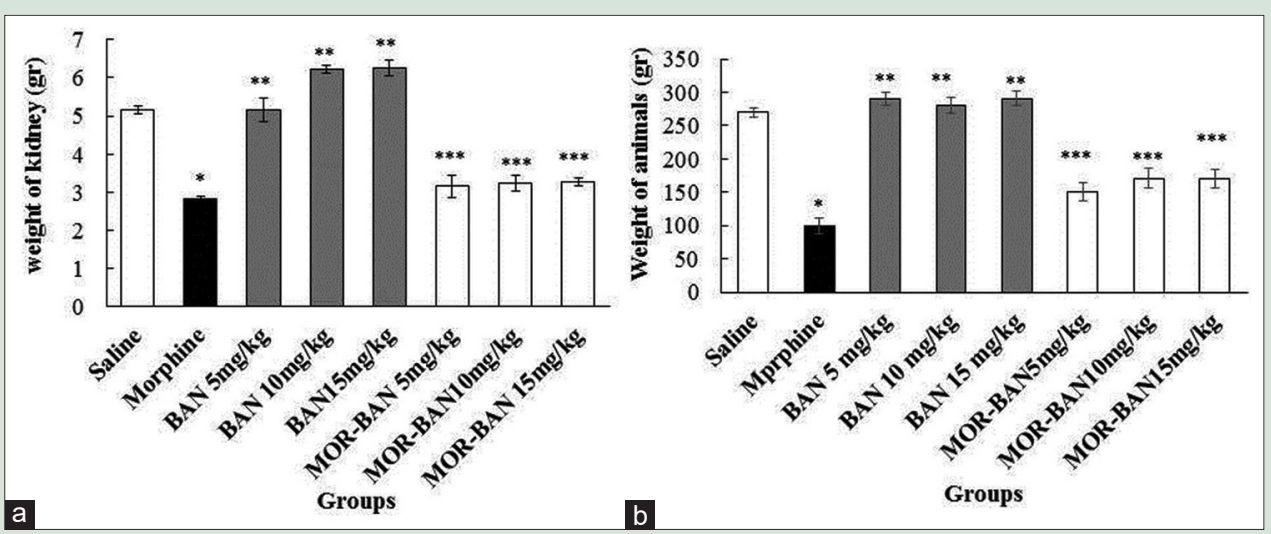

Figure 1: Effect of MOR, BAN, and BAN + MOR on weight of (a) kidney; (b) animals. *Significant different compared to Sal group ( $P<0.05)$. ${ }^{* *}$ Significant different compared to MOR group $(P<0.05)$. ${ }^{* *}$ Significant different compared to MOR group $(P<0.05)$. MOR: Morphine, BAN: Banisterine, Sal: Saline

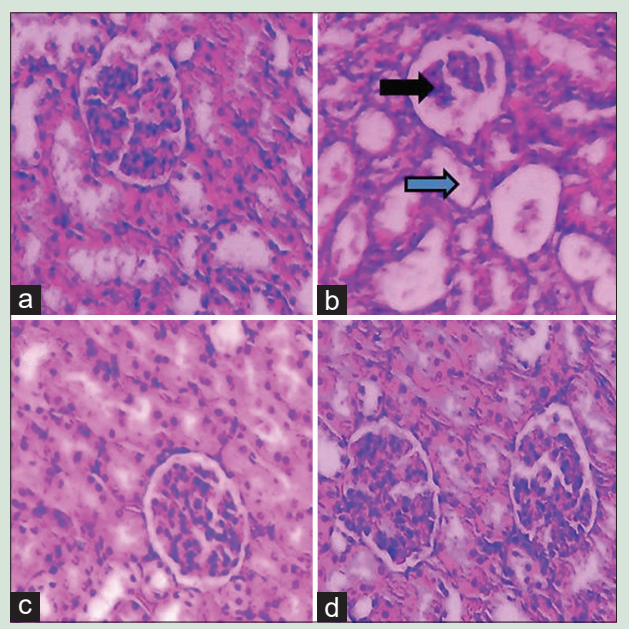

Figure 2: Histological changes in kidneys ( $H$ and $E, \times 400)$ : (a) normal kidney, Sal group; (b) MOR group, increased Bowman's capsule space and glomerular shrinkage (blue arrow) and increased distal and proximal tubule diameter (black arrow); (c) normal kidney, BAN $15 \mathrm{mg} / \mathrm{kg}$ group showing glomeruli with Bowman's capsule and distal and proximal tubules; (d) normal kidney structure in MOR + BAN $15 \mathrm{mg} / \mathrm{kg}$ group. MOR: Morphine, BAN: Banisterine, Sal: Saline

test. The value of $P<0.05$ was considered as statistically significant. All statistical analysis was done by SPSS software (version 16.0, SPSS Inc., Chicago, IL, USA).

\section{RESULTS}

\section{Total weight of animals and kidneys}

There was a clear trend of total weight and the kidney weight of treated animals as follows. The results of animal weathering offered a significant improvement in total weight of animal and weight of kidneys in all administered doses of $\operatorname{BAN}(5,10,15 \mathrm{mg} / \mathrm{kg})$ compared to the MOR $(P<0.05)$. No significant changes in the mean total weights of animal and kidney revealed in BAN treated animals in all doses $(5,10,15 \mathrm{mg} / \mathrm{kg})$ was revealed in comparison with the Sal group $(P>0.05)$. These two types of weighted variables were decreased significantly in animals treated with BAN + MOR in all doses (5, $10,15 \mathrm{mg} / \mathrm{kg})$ in comparison with the Sal group $(P<0.05)$. These variables were increased significantly in animals treated with BAN and BAN + MOR in all doses $(5,10,15 \mathrm{mg} / \mathrm{kg})$ in comparison with the
MOR $(P<0.05)$. Moreover, the effective dose of MOR was significantly decreased the mean total animal and kidney weight compared to the Sal group $(P<0.05)$ [Figure 1].

\section{Histological and morphometrical assessments}

The normal structure of kidney was detected in Sal and BAN treated groups. Histological observation revealed the disorganization of renal structure by MOR like an increase in the size of Bowman's capsule and distal and proximal tubules and also the count of glomeruli and intertubular bleeding were decreased. In Figure 2, the obvious data were presented indicating that the treatment in all doses $(5,10,15 \mathrm{mg} / \mathrm{kg})$ of MOR + BAN reduced the renal damage caused by MOR-related toxicity. According to morphometric concepts and the comparison to the Sal group, the mean diameter of the glomerulus tubule and the number of glomerular $(P<0.05)$ were improved and decreased, respectively. The BAN treatment increased the diameter of the glomeruli significantly $(P<0.05)$ while no significant effects on the number of glomeruli in all treatment groups $(5,10,15 \mathrm{mg} / \mathrm{kg})$ were detected compared to the Sal group $(P>0.05)$. The number of glomeruli in BAN + MOR treatments was significantly decreased $(P<0.05)$ while no significant influence on diameter of glomeruli in all treatment groups compared to the Sal group $(P>0.05)$ were detected. The histological alteration following BAN and BAN + MOR administration were clearly recognizable [Figure 3], indicating that the diameter of the glomeruli in the above-mentioned groups of MOR was decreased and the number of glomeruli was increased significantly compared to the MOR group $(P<0.05)$ [Figures 2 and 3 ].

\section{Biochemical markers}

According to the further biochemical trials, the MOR (with the dose of $2.5 \mathrm{ml} / \mathrm{kg}$ ) significantly increased the concentration of BUN and creatinine compared to the Sal group $(P<0.05)$ while the BUN and creatinine presented no significant changes in all doses $(5,10,15 \mathrm{mg} / \mathrm{kg})$ of BAN in comparison with Sal group $(P>0.05)$. According to Figure 4, the BUN and creatinine concentrations were increased significantly in all BAN + MOR groups $(5,10,15 \mathrm{mg} / \mathrm{kg})$ compared to the Sal group $(P<0.05)$ and were decreased significantly in all BAN and BAN $+\operatorname{MOR}(5,10$, $15 \mathrm{mg} / \mathrm{kg})$ groups compared to the MOR group $(P<0.05)$ [Figure 4].

\section{Nitrite oxide assay}

It is apparent that the average level of serum nitrite oxide was increased significantly in the MOR $(2.5 \mathrm{ml} / \mathrm{kg})$ group compared to the Sal group $(P<0.05)$ while it showed no significant changes in all BAN 


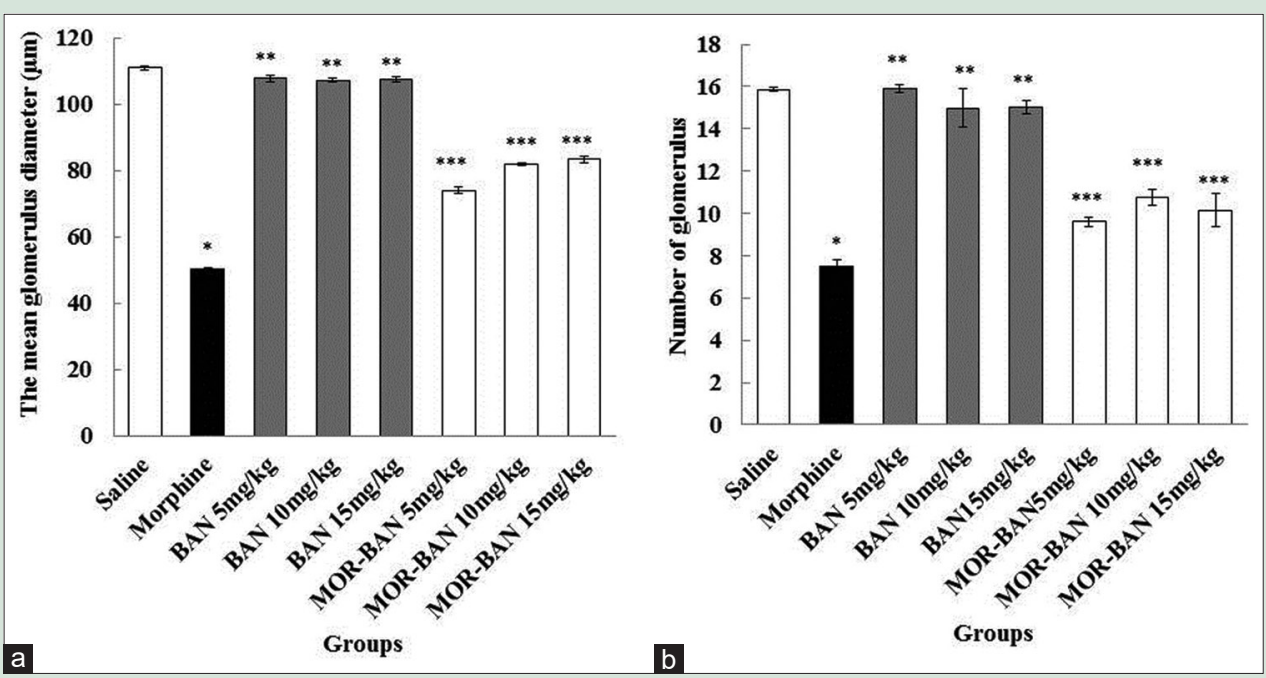

Figure 3: Correlation between treatment groups for (a) glomerular diameter; (b) glomeruli number. *Significant different compared to Sal group ( $P<0.05)$. **Significant different compared to MOR group $(P<0.05)$. ***Significant different compared to MOR group $(P<0.05)$. MOR: Morphine, BAN: Banisterine, Sal: Saline

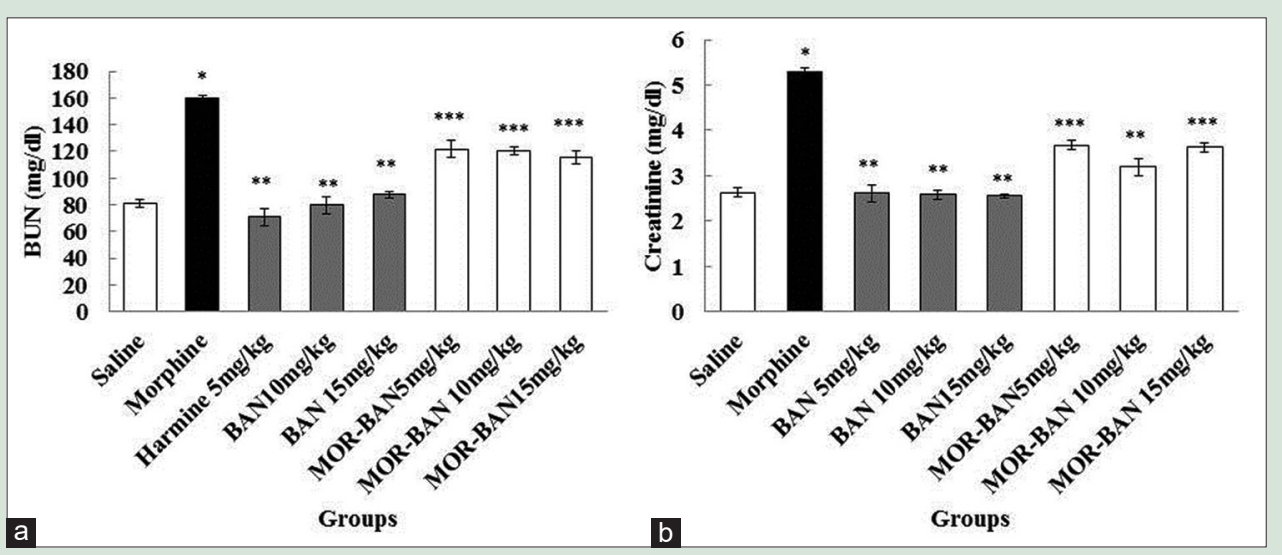

Figure 4: Effect of MOR, BAN and BAN + MOR on the mean kidney biochemical factors: (a) BUN (b) creatinine. *Significant different compared to Sal groups $(P<0.05)$. ${ }^{*}$ Significant different compared to MOR groups $(P<0.05)$. ${ }^{* *}$ Significant different compared to MOR group $(P<0.05)$. MOR: Morphine, BAN: Banisterine, BUN: Blood urea nitrogen, Sal: Saline

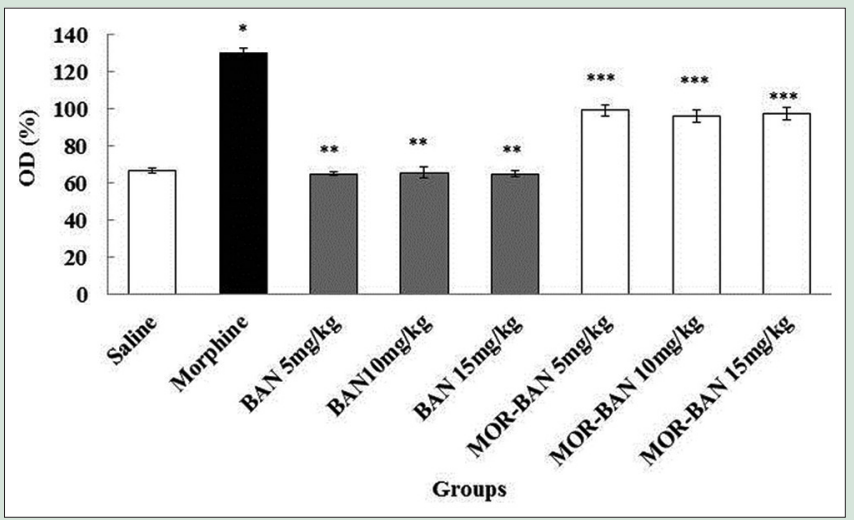

Figure 5: Effects of BAN, MOR and BAN + MOR on mean nitrite oxide levels. *Significant different compared to Sal group $(P<0.05) .{ }^{*}$ Significant different compared to MOR group $(P<0.05)$. ${ }^{* * *}$ Significant different compared to MOR group $(P<0.05)$. MOR: Morphine, BAN: Banisterine, Sal: Saline and BAN + MOR groups compared to the Sal group $(P>0.05)$. The mean serum level of nitrite oxide decreased significantly in all BAN and BAN + MOR groups $(5,10,15 \mathrm{mg} / \mathrm{kg})$ compared to the MOR group $(P<0.05)$ [Figure 5].

\section{Oxidative Stress and ferric reducing/antioxidant power assessments}

The level of renal MDA was significantly increased in the MOR group compared to the Sal group $(P<0.05)$. On the other hand, BAN decreased significantly the renal MDA level in all treatment groups $(5,10,15 \mathrm{mg} / \mathrm{kg})$ compared to the MOR group $(P<0.05)$. The renal MDA level was decreased significantly in all BAN + MOR groups $(5,10,15 \mathrm{mg} / \mathrm{kg})$ compared to the MOR group $(P<0.05)$. Similarly, MOR significantly reduced the renal tissue FRAP level in the MOR group compared to that of the Sal group $(P<0.05)$. BAN increased the FRAP level in the kidney in all BAN and BAN + MOR groups $(5,10,15 \mathrm{mg} / \mathrm{kg})$ compared to the MOR group $(P<0.05)$. In all groups of BAN, no significant changes in the renal tissue FRAPS and MDA levels were found compared to the Sal group $(P>0.05)$. Treatment with BAN + MOR significantly increased 

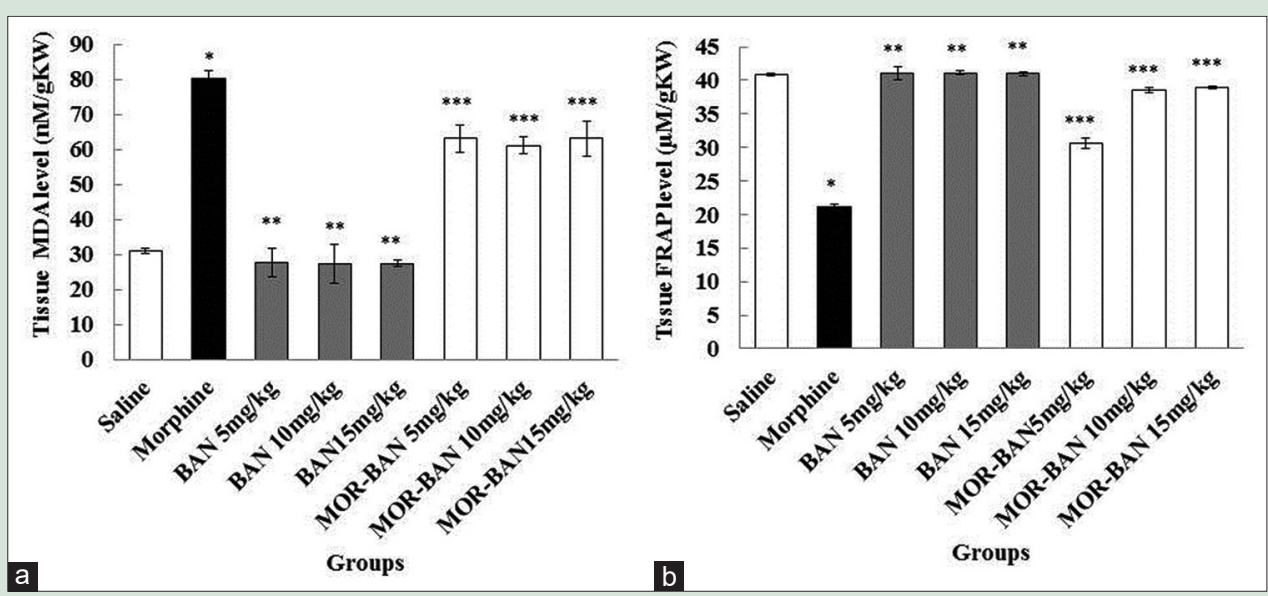

Figure 6: Comparison of MOR, Sal and BAN + MOR groups of: (a) kidney MDA level; (b) tissue FRAP level. *Significant different compared to Sal group ( $P<0.05)$ **Significant different compared to MOR group $(P<0.05)$. **Significant different compared to MOR group $(P<0.05)$. MOR: Morphine, BAN: Banisterine, MDA: Malondialdehyde, FRAP: Ferric reducing/antioxidant power

the renal MDA level $(P<0.05)$ while no significant effect on the renal tissue FRAPS level in all treatment groups $(5,10,15 \mathrm{mg} / \mathrm{kg})$ was found compared to the Sal group $(P>0.05)$ [Figure 6].

\section{DISCUSSION}

Chronic renal disease is a general health problem that affects all body organs including kidneys. A severe renal pain clinically alleviated by administration of MOR. Additionally, the MOR has pathogenesis effects on induction of renal deceases. ${ }^{[24]}$ BAN is an herbal alkaloid and a member of the Beta carboline family (extracted from P. harmala). This plant has various therapeutics effects in traditional medicine. ${ }^{[16]}$

The findings of the present survey will help us to understand whether the BAN extract has restorative effects on nephrotoxicity caused by increase MOR dosage in male Wistar rats. According to the results, there has been a gradual increase in total weight and kidney weight of MOR-receiving rats in comparison with the Sal group. Meanwhile, this incremental trend was observed in all doses of BAN + MOR groups compared to the MOR group. The stimulatory effect of MOR on release of dopamine, serotonin and $\gamma$-amino butyric acid in bloodstream and basic metabolism level of body and also inhibitory effects in appetite has been proved. ${ }^{[25]} \mathrm{A}$ recent study reported by Arany et al. supported this hypothesis that MOR decreases body weight. Arany et al. concluded that MOR with a high-fat diet regime decreased their body weight and BMI. ${ }^{[26]}$ It appears that MOR causes metabolism-induced generation of free radicals, lipid peroxidation, DNA destruction and cell membrane proteins, which finally causes cell damage. This phenomenon subsequently leads to weight of body and kidney loss. ${ }^{[1]}$ On the contrary, some papers confirm our conclusion about increasing the body weight by BAN consumption. For example, Hamden et al. found that $P$. harmala recovers weight loss in rats treated with thiourea. ${ }^{[27]}$ Scientists have seen that the BAN by binding to receptors such as mono amino oxidase, serotonin $2 \mathrm{~A}$ and kinase-dependent syncline improves the food absorption and total weights of body and kidney. ${ }^{[28]}$

The results obtained from the preliminary analysis of consumption of MOR alone revealed that MOR increases and decreases the glomerular diameter and number respectively. However, in MOR + BAN treated groups the glomerular diameter decreased and the number increased significantly compared to the MOR groups. These significant changes prove that the glomeruli are strongly sensitive to oxidative stress caused by MOR.${ }^{[7]} \mathrm{MOR}$ is a vigorous carcinogen that is oxidized into cotinine metabolites largely in the liver, kidneys, and lungs. Cotinine can run a critical role in the pathogenesis of tissue. ${ }^{[29]}$ MOR causes these changes with the application of cytochrome $\mathrm{P} 450$ and production of free radicals and oxidative stress in tissues. ${ }^{[3]}$ Lipid peroxidation and subsequent free radicals accumulation can change three-dimension structures of proteins and damage DNA and can induce apoptosis in renal tissue cells, vascular and tubular damage. ${ }^{[7,10]}$ The reduced average diameter and the number of glomeruli are found in renal function disorders. Jalili et al. in an experimental study reported that oxidative stress caused by mitochondrial degeneration leads to an increased concentration of cytoplasmic calcium. The alteration in biochemical hemostasis in cells, in addition to interfering with cytoskeleton arrangement and cellular metabolism, can even lead to initiation of intracellular enzymes activity like proteases, endonucleases and phospholipases. All cellular fluctuations as explained earlier resulting in necrosis of tubular epithelial cells. $^{[30]}$ The previously mentioned results correlate with those observed in the present study indicating that the BAN due to its antioxidant property largely neutralizes the oxidative stress effects of MOR. Thus the physiological number and diameter of glomeruli will be established, whereas the sole prescription of MOR increased BUN and creatinine. The combined application of MOR and BAN will significantly decrease the creatinine and BUN levels compared to the MOR group. The increased levels of BUN and creatinine may act as a signal to cause glomerular damage and disturb renal filtration capacity. ${ }^{[7,31]}$ The results of this study agree with the findings of other studies such as Osborne et al. which showed that the MOR increases the levels of BUN and creatinine. ${ }^{[10]}$ BAN can reduce the degenerative effects of MOR and prevent renal failure due to the reduction in the rate of oxidation and free radicals production. ${ }^{[32]}$

The level of nitrite oxide Evaluation indicated a significant increase in the level of nitrite oxide in MOR compared to Sal group. Prescription of BAN + MOR significantly decreased the nitrite oxide level. It is hypothesized that the MOR by discharge of noradrenaline in the paraventricular, amygdala and solitary nuclei stimulate the generation of nitrite oxide. ${ }^{[33]}$ The nitrite oxide by means of cellular mechanisms can enhance the excessive entry of calcium to cytosol which switches the physiological status of cells to the toxic form. ${ }^{[34]}$ Based on the evidences, excessive production of nitrite oxide and the increased iNOS and nNOS expression induce nephrotoxicity, nephritic and nephrotoxic diseases. ${ }^{[10]}$ NOS isoform is normally expressed in the kidney. If overexpression of NOS isoform occurs, the thickness of the distal tubules, proximal tubules and urine collecting tracts will increase. ${ }^{[35]}$ Antioxidants can diminish the rate of nitrite oxide production through disruption in the nitrite 
oxide system. ${ }^{[36]}$ As noted by El Madani et al. the BAN prescription significantly decreases the serum level of nitrite oxide in the rats treated by rotenone. ${ }^{[37]}$

The results of the current study reveal that the BAN as an antioxidant agent can lead to the alleviation of MOR -induced damage in renal tissue. Also, the BAN inhibits the induction of renal inflammation by reduction in serum level of nitrite oxide and increasing the total antioxidant capacity. The findings of the present study suggesting that the BAN can moderate lipid peroxidation and increase the activity of anti-oxidant in kidney. Therefore, there are several important changes by recruitment of anti-oxidant properties of BAN including an increase in FRAP and decrease in MDA levels.

\section{CONCLUSION}

The most prominent finding to emerge from this study is that the BAN recovers the nephrotoxicity condition caused by MOR application. Generally, these findings strongly support this hypothesis that the BAN acts as a valuable renal protective agent for individuals who have been exposed to MOR. This therapeutic effect is provided by antioxidant properties of BAN. Further investigations and experimentation into the molecular mechanism of BAN in renal MOR-toxicity are recommended.

\section{Acknowledgements}

We gratefully acknowledge the Research Council of Kermanshah University of Medical Sciences for the financial support of this study (Grant No: 9538).

\section{Financial support and sponsorship}

This work was supported by the Research Council of Kermanshah University of Medical Sciences.

\section{Conflicts of interest}

There are no conflicts of interest.

\section{REFERENCES}

1. Salahshoor MR, Roshankhah S, Hosseni P, Jalili C. Genistein improves liver damage in male mice exposed to morphine. Chin Med J (Engl) 2018;131:1598-604.

2. Salahshoor MR, Haghjoo M, Roshankhah S, Makalani F, Jalili C. Effect of thymoquinone on reproductive parameter in morphine-treated male mice. Adv Biomed Res 2018;7:18.

3. Fan Y, Chen Y, Zhang S, Huang M, Wang S, Li Y, et al. Morphine reverses the effects of 1-methyl-4-phenylpyridinium in PC12 cells through activating PI3K/Akt. Int J Neurosci 2019;129:30-5.

4. Kuo KL, Tarng DC. Oxidative stress in chronic kidney disease. Adaptive Med 2010;2:87-94

5. Therond P. Oxidative stress and damages to biomolecules (lipids, proteins, DNA). Ann Pharm Fr 2006;64:383-9.

6. Cyrus J, Shiva R, Reza SM. Falcaria vulgaris extract attenuates diabetes-induced kidney injury in rats. Asian Pac J Trop Biomed 2019;9:150-7.

7. Roshankhah S, Jalili C, Salahshoor MR. Protective effects of Petroselinum crispum on ischemia/reperfusion-induced acute kidney injury in rats. Physio Pharmacol 2019;23:129-39.

8. Costin D. Renal effects of deltamethrin induced intoxication in Carassius. Biotechnol Anim Husbandry 2007;23:399-404.

9. Fakurazi S, Rahman SA, Hidayat MT, Ithnin H, Moklas MA, Arulselvan P. The combination of mitragynine and morphine prevents the development of morphine tolerance in mice. Molecules 2013;18:666-81.

10. Osborne R, Joel S, Grebenik K, Trew D, Slevin M. The pharmacokinetics of morphine and morphine glucuronides in kidney failure. Clin Pharmacol Ther 1993;54:158-67.

11. Breyer-Pfaff U, Wiatr G, Stevens I, Gaertner HJ, Mundle G, Mann K. Elevated norharman plasma levels in alcoholic patients and controls resulting from tobacco smoking. Life Sci 1996;58:1425-32.
12. Liu WZ, Huang BW, You WJ, Hu P, Wang XH, Zhang JY, et al. Harmine enhances GABAergic transmission onto basoamygdala projection neurons in mice. Brain Res Bull 2018;137:294-300.

13. Mahmoudian M, Jalipour H, Salehian Dardashti P. Toxicity of Peganum harmala: Review and a case report. Iran J Pharm Therap 2002;1:1-10.

14. Mahmoudian M, Jalipour H, Salehian Dardashti P. Toxicity of Peganum harmala: Review and a case report. Iran J Pharmacol Ther 2002;1:1-0.

15. Salahshoor MR, Khazaei M, Jalili C, Keivan M. Crocin improves damage induced by nicotine on a number of reproductive parameters in male mice. Int J Fertil Steril 2016;10:71-8.

16. YH TS, Mak I, Dickens BF. Antioxidative properties of harmane and ß-carboline alkaloids. Biochem Pharmacol 1991;42:459-64.

17. Cao R, Peng W, Chen H, Ma Y, Liu X, Hou $X$, et al. DNA binding properties of 9 -substituted harmine derivatives. Biochem Biophys Res Commun 2005;338:1557-63.

18. Wang P, Alvarez-Perez JC, Felsenfeld DP, Liu H, Sivendran S, Bender A, et al. A high-throughput chemical screen reveals that harmine-mediated inhibition of DYRK1A increases human pancreatic beta cell replication. Nat Med 2015;21:383-8.

19. Yamazaki $Y$, Kawano $Y$. Inhibitory effects of herbal alkaloids on the tumor necrosis factor- $\alpha$ and nitric oxide production in lipopolysaccharide-stimulated RAW264 macrophages. Chem Pharm Bull (Tokyo) 2011;59:388-91.

20. Kobrin KL, Moody O, Arena DT, Moore CF, Heinrichs SC, Kaplan GB. Acquisition of morphine conditioned place preference increases the dendritic complexity of nucleus accumbens core neurons. Addict Biol 2016;21:1086-96.

21. Salman S, Idrees F, Pervaiz S, Shah FH, Badshah S, Usman M, et al. Evaluation of antimicrobial activities of harmine, harmaline, morphine and their complexes. Pak J Pharm Sci 2016;29:1317-20

22. Salahshoor MR, Roshankhah S, Motavalian V, Jalili C. Effect of harmine on nicotine-induced kidney dysfunction in male mice. Int J Prev Med 2019;10:97.

23. Najafi H, Owji SM, Kamali-Sarvestani E, Moosavi SM. A1 -Adenosine receptor activation has biphasic roles in development of acute kidney injury at 4 and $24 \mathrm{~h}$ of reperfusion following ischaemia in rats. Exp Physiol 2016;101:913-31.

24. Jain G, Jaimes EA. Morphine signaling and progression of chronic kidney disease in smokers. Biochem Pharmacol 2013;86:1215-23.

25. Salahshoor M, Mohamadian S, Kakabaraei S, Roshankhah S, Jalili C. Curcumin improves liver damage in male mice exposed to nicotine. J Tradit Complement Med 2016;6:176-83.

26. Arany I, Hall S, Reed DK, Reed CT, Dixit M. Morphine enhances high-fat diet-induced oxidative stress in the kidney. Morphine Tob Res 2016;18:1628-34.

27. Hamden K, Masmoudi H, Ellouz F, ElFeki A, Carreau S. Protective effects of Peganum harmala extracts on thiourea-induced diseases in adult male rat. J Environ Biol 2008;29:73-7.

28. Fortunato JJ, Réus GZ, Kirsch TR, Stringari RB, Fries GR, Kapczinski F, et al. Effects of $ß$-carboline harmine on behavioral and physiological parameters observed in the chronic mild stress model: Further evidence of antidepressant properties. Brain Res Bull 2010;81:491-796.

29. Yildiz D, Liu YS, Ercal N, Armstrong DW. Comparison of pure morphine-and smokeless tobacco extract-induced toxicities and oxidative stress. Arch Environ Contam Toxicol 1999;37:434-9.

30. Jalili C, Salahshoor MR, Naderi T. The effect of hydroalcoholic extract of P. crispum on sperm parameters, testis tissue and serum nitric oxide levels in mice. Adv Biomed Res 2015;4:40

31. Esfandiari E, Roshankhah S, Mardani M, Hashemibeni B, Naghsh E Kazemi $\mathrm{M}$, et al. The effect of high frequency electric field on enhancement of chondrogenesis in human adipose-derived stem cells. Iran J Basic Med Sci 2014; 17:571-6.

32. Zetler G, Back G, Iven H. Pharmacokinetics in the rat of the hallucinogenic alkaloids harmine and harmaline. Naunyn Schmiedebergs Arch Pharmacol 1974:285:273-92.

33. Zhao R, Sharp BM. Morphine-induced norepinephrine release in hypothalamic paraventricular nucleus and amygdale is mediated by $\mathrm{N}$-methyl-D-aspartate receptors and nitric oxide in the nucleus tractus solitarius. J Pharmacol Exp Ther 2007;320:837-44.

34. Calabrese V, Mancuso C, Calvani M, Rizzarelli E, Butterfield DA, Stella AM. Nitric oxide in the central nervous system: Neuroprotection versus neurotoxicity. Nat Rev Neurosci 2007;8:766-75.

35. Sharma S. Nitric oxide and the kidney. Indian J Nephrol 2004;14:77-84.

36. Salahshoor MR, Jalili C, Roshankhah S. Can royal jelly protect against renal ischemia/reperfusion injury in rats? Chin J Physiol 2019;62:131-7.

37. El Madani MA, Attia A, El-Shenawy S. Neuropharmacological effects of naringenin harminee and adenosine on Parkinsonism induced in rats. Der Pharmacia Lettre 2016;8:45-8. 